\title{
Development of the Method for Predicting and Calculating the Operation of Sorption Systems for Cleaning the Generator Gas based on Dolomite Use. Part II
}

\author{
${ }^{1}$ Malko M.V., ${ }^{2}$ Vasilevich S.V., ${ }^{3}$ Mitrofanov A.V., ${ }^{3}$ Mizonov V.E. \\ ${ }^{1}$ Institute of Power Engineering of the National Academy of Sciences of Belarus, Minsk, \\ Republic of Belarus, \\ ${ }^{2}$ Belarusian State Academy of Aviation, Minsk, Republic of Belarus, \\ ${ }^{3}$ Ivanovo State Power Engineering University, \\ Ivanovo, Russian Federation
}

\begin{abstract}
At present, instead of a direct combustion of solid fuel, its thermochemical conversion is extensively used to produce a generator gas. The use of this technology is connected with the need for gas purification. One of the promising and widely spread sorbents for the purification of the generator gas is dolomite, whose particles compose the active component of the bed filters. Forecasting the technological characteristics of the functioning of the bed filters of a various design is an extremely urgent task. The objective of the study is to develop a method for forecasting and calculating the operation of sorption systems for purification of the generator gas based on dolomite. It is achieved by constructing and verifying a mathematical model of the functioning of the bed sorption filter with a radial-axial flow pattern of the generator gas through the dolomite filling. The Markov chains theory of a mathematical apparatus is used to design the one-dimensional mathematical model of the process with discrete space and time. The main recurrent balance ratio is formed at each calculation step taking into account the current characteristics of the process, which makes the model nonlinear. The significance of the research is that an approach to the problem of increasing the reliability of the description and reliability of forecasting technological processes in a bed filter was proposed based on the construction of mathematical models of these processes, in which the filter is considered as a system with distributed characteristics, and the calculation was based on local exchange potentials between particles and gas.
\end{abstract}

Keywords: generator gas, dolomite, thermal decomposition, sorbent, thermogravimetric analysis, kinetics of decomposition, generator gas purification system.

DOI: https://doi.org/10.52254/1857-0070.2021.4-52.04

UDC: $\mathbf{5 4 3 . 5 7 3}$

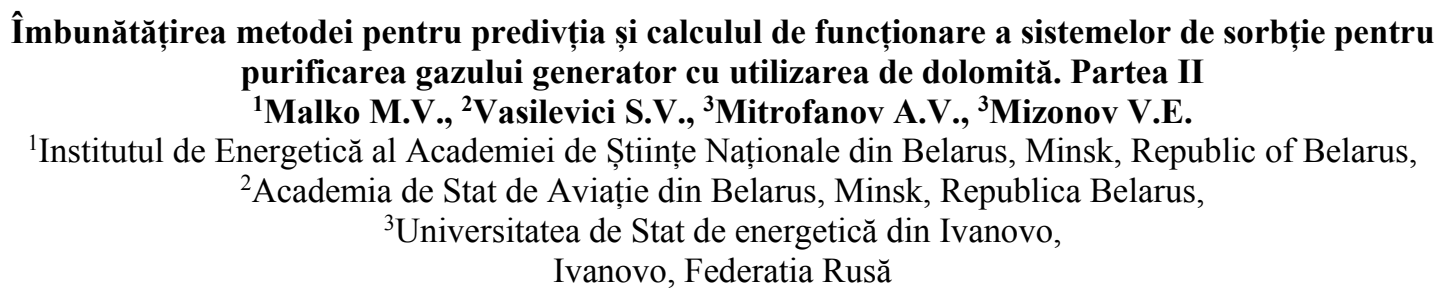

Rezumat. Actualmente, în loc de arderea directă a combustibilului solid, conversia termochimică a acestuia este utilizată pe scară largă pentru a obține gazul de generator. Implementarea unei asemenea tehnologii este în mod indisolubil legată de necesitatea efectuării purificării gazului. Unul din cele mai de perspectivă și răspândit pe larg sorbent pentru purificarea gazului de generator este dolomitul, particulele căruia alcătuiesc componenta activă a filtrelor în straturi. Prognozarea caracteristicilor tehnologice de funcționare a filtrelor în straturi de diversă interpretare constructivă este un subiect de maximă actualitate. Scopul lucrării este de a îmbunătăţi metoda de predicție și calcul a funcționării sistemelor de sorbție pentru curățarea gazului generator în bază de dolomit. Acest obiectiv este atins prin construirea și verificarea unui model matematic de funcţionare a unui filtru de sorbție în strat cu o schemă radial-axială de mișcare a gazului generator prin rambleul de dolomit. Cel mai semnificativ rezultat al studiului este acela că se realizează identificarea parametrică a modelului propus, ceea ce îi oferă proprietăţi predictive, iar adecvarea modelului este verificată prin compararea previziunilor obținute cu rezultatele unui experiment firesc, principial independent de schema de modelare și în faptul că se propune o abordare asupra soluționării problemei creșterii credibilităţii descrierii și a fiabilităţii proceselor tehnologice de predicție într-un filtru stratificat, bazată pe construcția unor modele matematice ale acestor

(C) Малько М.В., Василевич С.В.,

Митрофанов А.В., Мизонов В.Е., 2021 
procese, în care filtrul este considerat un sistem cu caracteristici distribuite, iar calculul se realizează folosind potențialele de schimb între particule și gaz.

Cuvinte-cheie: gaz generator, dolomit, sorbent, teoria lanțurilor Markov, model de celule, sistem de purificare a gazului generator.

\section{Совершенствование метода прогнозирования и расчета работы сорбционных систем очистки генераторного газа на основе доломита. Часть II \\ ${ }^{1}$ Малько М.В., ${ }^{2}$ Василевич С.В., ${ }^{3}$ Митрофанов А.В., ${ }^{3}$ Мизонов В.Е. \\ ${ }^{1}$ Институт энергетики НАН Беларуси, Минск, Республика Беларусь, ${ }^{2}$ Белорусская государственная академия авиации, Минск, Республика Беларусь, ${ }^{3}$ Ивановский государственный энергетический университет, \\ Иваново, Российская Федерация}

Аннотация. В настоящее время вместо прямого сжигания твердого топлива достаточно широко используется его термохимическая конверсия с целью получения генераторного газа. Внедрение подобной технологии неразрывно связано с необходимостью проведения газоочистки. Одним из перспективных и широко распространенных сорбентов для очистки генераторного газа является доломит, частицы которого составляют активный компонент слоевых фильтров. Прогнозирование технологических характеристик функционирования слоевых фильтров различного конструктивного исполнения является крайне актуальной задачей. Цель данной работы состоит в совершенствовании метода прогнозирования и расчета работы сорбционных систем очистки генераторного газа на основе доломита. Поставленная цель достигается за счет построения и верификации математической модели функционирования слоевого сорбционного фильтра с радиально-аксиальной схемой движения генераторного газа через засыпку доломита. Для построения одномерной математической модели процесса с дискретным пространством и временем использован математический аппарат теории цепей Маркова. Основное рекуррентное балансовое соотношение при этом формируется на каждом расчетном шаге с учетом текущих характеристик процесса, что делает модель нелинейной. Наиболее существенным результатом исследования является то, что выполнена параметрическая идентификация предложенной модели, которая наделяет ее прогностическими свойствами, а также выполнена проверка адекватности модели через сопоставление получаемых прогнозов с результатами натурного принципиально независимого от схемы моделирования эксперимента. Значимость результатов исследования состоит в том, что предложен подход к решению проблемы повышения достоверности описания и надежности прогнозирования технологических процессов в слоевом фильтре, основанный на построении математических моделей этих процессов, в которых фильтр рассматривается как система с распределенными характеристиками, а расчет ведется по локальным потенциалам обмена между частицами и газом.

Ключевые слова: генераторный газ, доломит, сорбент, теория цепей Маркова, ячеечная модель, система очистки генераторного газа.

\section{ВВЕДЕНИЕ}

$\begin{array}{ccrr}\text { В } & \text { настоящее } & \text { время } & \text { одним } \\ \text { доминирующих } & \text { направлений } & \text { развития }\end{array}$ твердотопливной энергетики стал частичный отказ от прямого сжигания топлива и развитие технологий его термохимической конверсии [1-3]. Подобные проекты, как правило, предполагают получение генераторного газа, при этом эффективность всего процесса существенно ограничивается отсутствием достаточно изученных и, соответственно, хорошо прогнозируемых способов очистки генераторного газа [3-4] при высокой стоимости очистных систем (до 15-20 \% от общей цены строительства ТЭС с парогазовой установкой [3]).

Аппаратно-процессуальное оформление сорбционных систем газоочистки, так же как и вопросы, связанные с расчетом и прогнозированием параметров их работы, неразрывно связано с выбором материаласорбента. Известно, что термическое разложение карбоната кальция $\left(\mathrm{CaCO}_{3}\right)$ начинается при достижении материалом температур порядка $800^{\circ} \mathrm{C}$ [5], что позволяет при более низких температурах использовать обратную реакцию соединения оксида кальция с углекислым газом в системах газоочистки для связывания $\mathrm{CO}_{2}$.

Как было отмечено ранее, ввиду дефицита чистых пород карбоната кальция перспективным сорбентом для очистки генераторного газа является доломит, который содержит и другие химические соединения.

Традиционно доломитом называют породу, в состав которой входит двойная карбонатная соль кальция и магния $\left(\mathrm{CaMg}\left(\mathrm{CO}_{3}\right)_{2}\right)$ с 
примесями карбоната кальция $\left(\mathrm{CaCO}_{3}\right)$, оксидов железа, алюминия, кремния, марганца и некоторых других соединений [69].

В первой части настоящего исследования был выполнен комплексный анализ состава доломитового сырья, кинетики термического разложения одиночных частиц доломита различных размеров. Были определены значения кинетических параметров этого процесса в зависимости от температурного режима и размера (массы) исследуемых образцов. В результате были предложены зависимости для прогнозирования кинетических параметров брутто-реакции от размера исходной частицы и температуры обработки. То есть были рассмотрены процессы, приводящие к преобразованию доломитового сырья в активный сорбент (отожженный доломит).

Таким образом, на наш взгляд, первая часть работы определяет достаточный уровень знания о процессе формирования или регенерации сорбента, а поэтому может служить отправной точкой для дальнейшего совершенствования методов расчета систем очистки пиролизных газов.

В основе работы фильтров лежит химическое взаимодействие продуктов (CaO и $\mathrm{MgO}$ ) термической диссоциации доломита с газогенераторным газом. Оксиды кальция и магния связывают диоксид углерода $\left(\mathrm{CO}_{2}\right)$, содержащиеся в газогенераторном газе, образуя карбонаты кальция и магния соответственно $\left(\mathrm{CaCO}_{3}\right.$ и $\left.\mathrm{MgCO}_{3}\right)$. Соответствующие реакции, объясняющие механизм очистки генераторного газа, имеют следующий вид [5]:

$$
\begin{aligned}
& \mathrm{MgO}+\mathrm{CO}_{2} \stackrel{=}{\longleftrightarrow} \mathrm{MgCO}_{3}, \\
& \mathrm{CaO}+\mathrm{CO}_{2} \stackrel{=}{\longleftrightarrow} \mathrm{CaCO}_{3} .
\end{aligned}
$$

Кинетика реакций (1) - (2) исследована в достаточной степени [10-14], однако, при работе в качестве сорбента зерна доломита обычно организуются в неподвижный слой, через который фильтруется генераторный газ. Описание процесса в слоевом фильтре требует поиска инструментов, при помощи которых будет показан характер распределения полей температур и концентраций (в частном случае, может оказаться, что при некоторых геометрических размерах фильтр может быть рассмотрен как объект с идеальным смешением свойств).

Выбор математических инструментов для декомпозиции слоя на некоторые локальные представительные объемы может быть выполнен при помощи разных математических инструментов. Это может быть подход Эйлера, подход Лагранжа или комбинированные модели на основе этих подходов [15-16].

Подобные подходы, предельно детализируя структуру объекта, с формальных позиций являются, по-видимому, наиболее непротиворечивыми в качественном отношении. Однако количество параметров в подобных моделях велико, что делает часто невозможной их однозначную идентификацию. В связи с этим получают достаточно широкое распространение в инженерной практике искусственные подходы, которые исходят не из рассмотрения локальных областей условно бесконечно малого размера, а введении в рассмотрение некоторого компромиссного («мезоскопического») представительного размера [17]. При этом связи между введенными в рассмотрение локальными объемами могут устанавливаться на основе различных подходов: с использованием математического аппарата теории цепей Маркова [18], дискретных аналогов уравнения Больцмана [19], теории клеточных автоматов [20] и др.

Цель данной работы состоит в экспериментальном исследовании сорбционного слоевого фильтра на основе доломита с радиально-аксиальным движением потока генераторных газов и разработке математической модели этого процесса, которая позволяла бы рассмотреть фильтр как объект с распределенными характеристиками.

\section{МАТЕРИАЛЫ И МЕТОДЫ}

В первой части работы было дано достаточно детальное описание пиролизного реактора и системы очистки пиролизного газа. На рис. 1а приводится принципиальная схема этой системы, а на рис. 16 и 1в показаны фотография самого слоевого фильтра и перфорированной кассеты для загрузки доломитового материала, на исследование функционирования которого направлена данная часть работы. Необходимо 
отметить, что основные габаритные размеры фильтра приведены в первой части работы.

Фильтр представляет собой слоевой реактор, состоящий из трех цилиндрических емкостей с различными диаметрами, которые монтируются соосно. Внутренняя и средняя цилиндрические поверхности перфорированы с количеством отверстий 246 отверстий на внутренней поверхности и 2050 на средней. Диаметр отверстий перфорации - 4 мм. В кассету (объем между перфорированными цилиндрами) помещается сорбент (обожженный доломит). Высота засыпки составляет 500 мм, толщина цилиндрического слоя - 120 мм. В полость внутреннего цилиндра помещается вставка (обтекатель) в виде тела вращения конической формы.

Температура пиролизного газа на входе в камеру поддерживалась равной $650^{\circ} \mathrm{C}$, давление 1 атм, а расход составлял 0,025 кг/ч. Температура в реакционной камере регистрировалась с помощью термопары 3 и измерителя-регулятора температур «Сосна002» 4, обеспечивающих возможность работы при измерении температур до 1373 К с точностью измерения до $\pm 0,01 \kappa$.
Состав пиролизного газа на выходе из газогенератора измерялся с помощью газоанализатора «Дэкос» 5, позволяющего измерять состав газовых смесей, температуру, давление, скорость течения, объемный расход с относительной погрешностью концентраций отдельных компонентов $\pm 5 \%$, температуры \pm 2 , давления \pm 1 отн. $\%$, скорости потока $\pm 0,01$ м/с. Расход пиролизных газов измерялся при помощи ротаметра типа РМ.

В опытах образцы отожженного доломита выдерживались при заданной постоянной температуре. Через образцы пропускался пиролизный газ с фиксированным содержанием $\mathrm{CO}_{2}$. Macca образцов измерялась через определенные промежутки времени на электронных весах Stohl c относительной погрешностью $2 \%$.

В эксперименте фиксировалась степень завершенности суммарной реакции (валового результата протекания реакций (1)-(2)), которая определялась по общему приросту массы навески материала в кассете, выдерживаемого при заданной температуре.

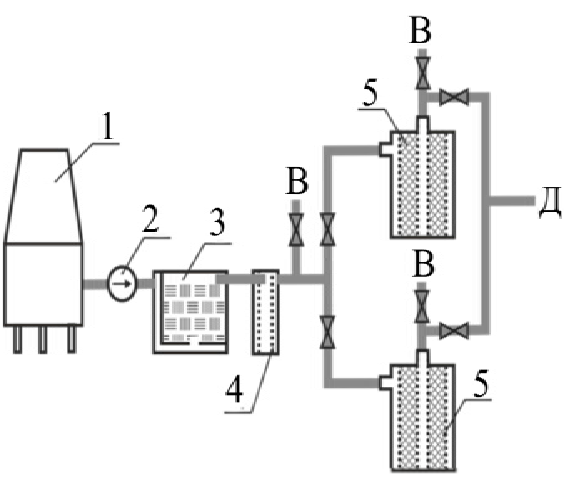

a)

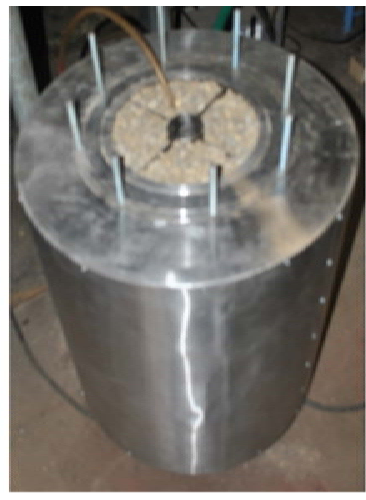

б)

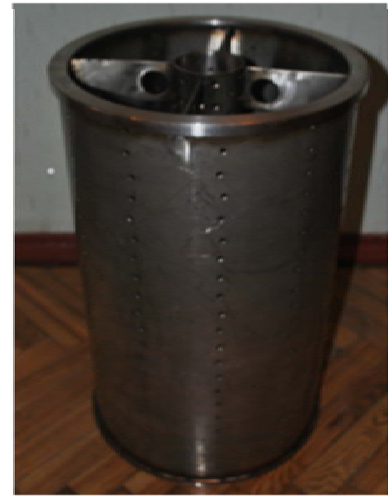

B)

1 - газогенератор; 2 - скруббер Вентури с насосом, водоотделительным ящиком и сточной емкостью;

3 - фильтр тонкой очистки ø700 мм; 4 - контрольный фильтр ø300 мм; 5 - реактор очистки топливного газа с радиально-аксиальныл движением потока с применением доломита; $B$ - коллектор, ведущий к факельную установку с регулирующим вентилем; Д-коллектор, ведущий к газопоршневому двигателю с электрогенератором.

Рис. 1. Принципиальная схема пиролизного реактора и подключения слоевых фильтров очистки пиролизных газов (а), фотографии общего вида экспериментального образца слоевого фильтра очистки с радиально-аксиальным движением газов (б) и перфорированной кассеты (в). ${ }^{1}$

Для построения адекватной модели процесса необходим некоторый объем априорного знания о процессе.

В данном случае для ответа на вопрос о характере движения газового агента внутри фильтра были проведены следующие исследования, проведенные на специально созданном лабораторном стенде с использованием экспериментального образца реактора очистки с радиально-аксиальным движением газов, описание которого приведено выше. Кассета была заполнена

${ }^{1}$ Appendix 1 
доломитовой крошкой осколочной формы, полученной из доломитового щебня плотностью 2.85 г $/ \mathrm{cm}^{3}$.

Эквивалентный диаметр частиц в навеске составил 2.7 мм. Схема установки представлена на рис. 2.

Равномерность течения воздуха по засыпке доломита определялась по распределению потерь давления в рабочей камере реактора. Трубки отбора статического давления вводились в рабочую камеру реактора по наружному, среднему и внутреннему диаметрам (по четыре датчика для каждой радиальной позиции). Измерение избыточного давления осуществлялось в различных точках реактора на высоте $0.1 \mathrm{~L}$, $0.5 \mathrm{~L}$ и $0.9 \mathrm{~L}$ (L - общая высота засыпки).

В качестве газового агента для изучения гидравлических характеристик слоя доломита в реакторе очистки топливного газа с радиально-аксиальным движением потока, использовался атмосферный воздух. Для измерения объемного расхода газового агента на входе в реактор использовался ротаметр типа РМ, предназначенный для измерения объемного расхода плавно меняющихся однородных потоков чистых и слабозагрязненных жидкостей и газов с дисперсными включениями инородных частиц.

На рис. 3 представлена схема расположения датчиков манометра $(\mathrm{a}$, б) и некоторые результаты экспериментального исследования характера распределения статического давления газа (в).

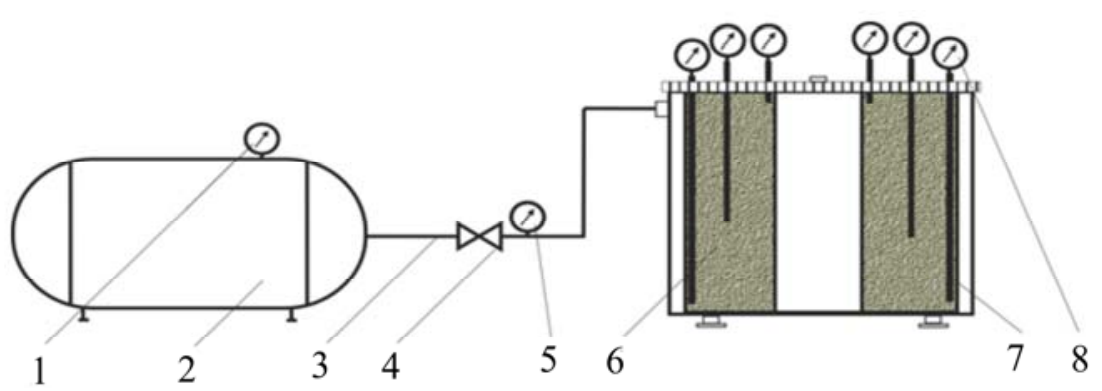

1 -манометр компрессора; 2 - компрессор; 3 - подающий трубопровод; 4 -регулировочный вентиль; 5

- манометр контроля давления на входе в аксиально-радиальный реактор; 6-аксиально-радиальный реактор; 7 -измерительные трубки; 8 -манометры измерительных трубок

Рис. 2. Общая схема лабораторного стенда по изучению гидравлических характеристик слоя доломита в слоевом фильтре. ${ }^{2}$

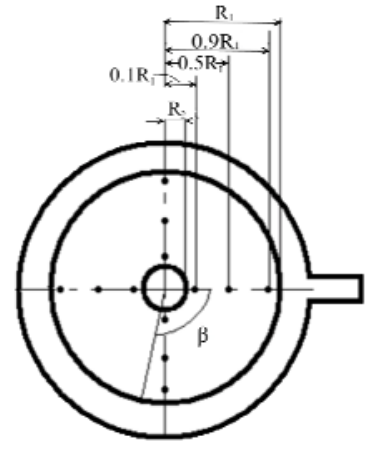

a)

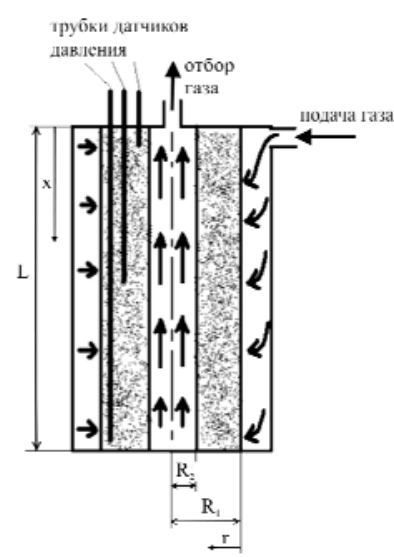

б)

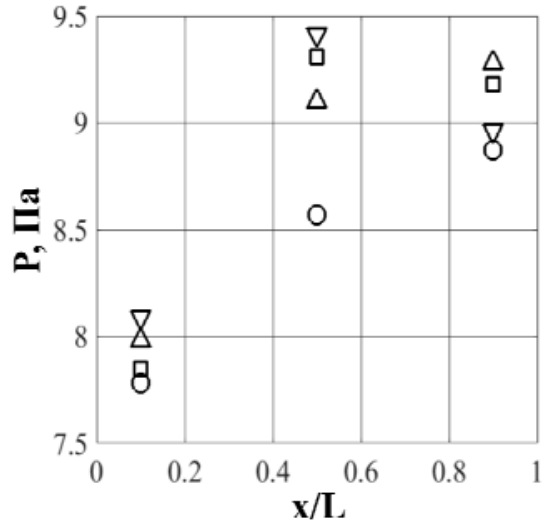

B)

маркеры - экспериментальные значения: $\square-$ дя $\beta=0$, о- для $\beta=90 ; \Delta-\partial л я ~ \beta=180 ; \nabla-\partial л я ~ \beta=270$.

Рис. 3. К описанию эксперимента по измерению статистического давления в слое: схема радиальноаксиального реактора в разрезе с точками замера статического давления ((a) - вид сверху; (б) - вид сбоку), (в) - результаты измерений. ${ }^{3}$

${ }^{2,3}$ Appendix 1 
Как показывают представленные результаты, величина давления слабо зависит от азимутального угла $\beta$ расположения датчика, из чего можно сделать вывод, что подаваемый газовый агент распределяется достаточно равномерно по боковой поверхности кассеты. Также находит подтверждение то обстоятельство, что проходящий через реактор газ равномерно распределяется по высоте реактора, на что указывает слабая зависимость давления от

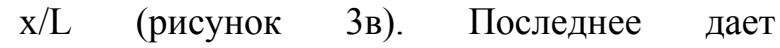
возможность принять для описания выбранной конструкции реактора очистки с аксиально-радиальным направлением движения газов одномерную математическую модель.

Расчетная схема моделирования поясняется на рисунке 4. Рассматривается одномерная осесимметричная задача, в которой в качестве домена выбран цилиндрический слой фиксированной толщины $\Delta \mathrm{r}$.

Рассматривается случай фильтрации газовой среды с непрерывным по мере движения исчезновением компонента газа в результате химической реакции с частицами дисперсной среды. С точки зрения представлений механики сплошной среды математической моделью такого процесса служит обобщенный случай уравнения неразрывности [21]:

$$
\frac{\partial \rho}{\partial t}+\frac{\partial\left(\rho v_{r}\right)}{\partial r}-J=0
$$

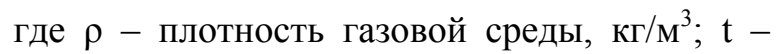
время процесса, с; $\mathrm{V}_{\mathrm{r}}$ - радиальная скорость движения газовой среды, м/c; r - радиальная координата; J - объемная плотность потока массы при межфазном взаимодействии газчастицы, $\left[\kappa \Gamma \cdot \mathrm{c}^{-1}\right] / \mathrm{M}^{3}$.

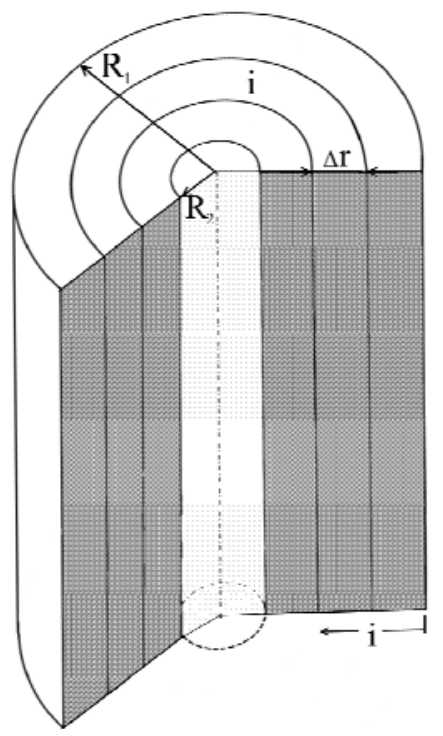

Рис. 4. Расчетная схема математической ячеечной модели. ${ }^{4}$

Для построения простой математической модели процесса уравнение (3), на наш взгляд, не вполне удобно. В то же время его анализ полезен с точки зрения обозначения тех явлений, которые должны быть приняты во внимание при математической формализации задачи в виде разностной схемы, а также определения тех допущений, которые будут приняты при моделировании.

Так, первое слагаемое уравнения (3) описывает изменение во времени объемного содержания газовой фазы в представительном объеме системы. В дальнейшем будем полагать, что продвижение газовой фазы в радиальном направлении не предполагает его сжатия $(\partial \rho / \partial t=0)$. Такое допущение позволяет считать, что результатом перемещения некоторого объема газа из рассматриваемой ячейки в следующую является изменение его скорости. Таким образом, полагается наличие установившегося поля скоростей газа по ячейкам (схема нумерации ячеек проиллюстрирована на рис. 4). Внутри $i-$ го цилиндрического слоя скорость газа $U$ может быть определена как:

$$
U_{i}=(1-\varepsilon) \cdot U_{0} \cdot\left(F_{1} / F_{i}\right),
$$


где $\varepsilon-$ порозность засыпки доломита $(\varepsilon=0.7) ; U_{0}-$ скорость газа в первой ячейке, м/c; $F$-площадь наружной боковой поверхности ячейки (цилиндрического слоя), $\mathrm{M}^{2}$.

Второе слагаемое в уравнении (3) описывает конвективный поток газа сквозь сыпучий материал. Приняв допущение о движении газа в режиме идеального вытеснения, можно считать справедливыми следующие соотношения. Доля газа, удаляемая из ячейки за малый конечный промежуток времени $\Delta \mathrm{t}$ равна

$$
p_{i}=\frac{U_{i} \cdot F_{i} \cdot \Delta t}{V_{i}},
$$

где $V_{i}$-объем ячейки, м ${ }^{3}$ (вычисляется как объем цилиндрического слоя толщиной $\Delta r)$.

Применяя математический аппарат теории цепей Маркова [22] для описания продвижения со временем радиального потока газа запишем основной оператор модели (переходную матрицу) Р. Для случая потока с идеальным вытеснением переходная матрица примет вид:

$\Delta r$

$$
\mathbf{P}=\left[\begin{array}{cccccc}
1-p_{1} & 0 & \ldots & 0 & 0 & 0 \\
p_{1} & 1-p_{2} & \ldots & 0 & 0 & 0 \\
0 & p_{2} & \ldots & 0 & 0 & 0 \\
\ldots & \ldots & \ldots & \ldots & \ldots & \ldots \\
0 & 0 & \ldots & 1-p_{n-1} & 0 \\
0 & 0 & \ldots & p_{n-1} & 1-p_{n}
\end{array}\right] .
$$

Тогда, продвижение массы газовой фазы за малый конечный промежуток времени $\Delta \mathrm{t}$ может быть описано при помощи следующего рекуррентного матричного соотношения [22]:

$$
\mathbf{S}^{k+1}=\mathbf{P} \cdot \mathbf{S}^{k}+\mathbf{G}^{k}
$$

где $\mathbf{S}$ - вектор, характеризующий состояние газовой фазы в аппарате (каждый элемент вектора - это масса газовой фазы в соответствующей ячейке); вектор $\mathbf{G}$ - вектор источников газовой фазы, имеющий единственный ненулевой элемент (при $\mathrm{i}=1$ ), значение которого равно массе газа, поступающей в эту ячейку за время одного временного перехода $\Delta t ; \mathrm{k} \quad-$ номер рекуррентного расчетного шага, на котором фиксируется состояние системы.

Таким образом, соотношение (7) позволяет описать состояние системы в дискретные моменты времени $t_{k}=(k-1) \Delta t$.

C учетом принятых допущений переходная матрица Р не зависит от времени (и номера рекуррентного шага), так как значения ее элементов рассчитываются с учетом принятого ранее допущения о существовании стационарного поля скоростей, определяемого зависимостью (4).

Однако, продолжая анализ уравнения неразрывности, записанного в форме (3), необходимо отметить, что третье слагаемое J в нем отвечает за потоки вещества, связанные с межфазным взаимодействием. В контексте настоящей работы и предлагаемой расчетной схемы этот процесс трактуется как наличие стокового слагаемого, которым дополняется балансовое уравнение (7). Вводя соответствующее обозначение, таким образом, можно записать.

$$
\mathbf{S}^{k+1}=\mathbf{P} \cdot \mathbf{S}^{k}-\mathbf{M}^{k}+\mathbf{G}^{k}
$$

где $\mathbf{M}$ - вектор масс газа (фактически масса поглощаемого $\mathrm{CO}_{2}$ ), поглощаемых за время $\Delta \mathrm{t}$ в ячейках. Необходимо отметить, что вектор М имеет ту же размерность, что и вектор $\mathbf{S}(n \times 1)$. Также видно, что вектор $\mathbf{M}$ зависит от рекуррентного расчетного шага, так как, очевидно, реакционная способность доломита изменяется по мере реализации реакции.

Вектор потери газовой фазой массы М зависит от того, какое количество $\mathrm{CO}_{2}$ будет поглощено за счет протекания реакций (1)-(2) за время k-о рекуррентного расчетного шага. Скорость этих реакций может быть описана с помощью известных кинетических зависимостей.

В инженерных расчетах принято рассматривать реакции (1)-(2) как формальный процесс термопреобразования материала [23], скорость которого может быть описана с помощью уравнения следующего вида [24]:

$$
\frac{d x}{d t}=k_{p} \cdot\left(1-\frac{x}{x_{u}}\right)^{n},
$$


где $x$ - степень завершенности формальной реакции, $k_{p}$-зависящая от температуры константа скорости процесса, $\mathrm{c}^{-1}$.

Степень завершенности суммарной реакции формально определяется как:

$$
x=\frac{\mu_{C a O}}{\mu_{C O}} \cdot\left(\frac{m_{t}-m_{0}}{c \cdot m_{0}}\right),
$$

где $\mu_{\mathrm{CaO}}$ и $\mu_{\mathrm{CO} 2}$ - молекулярные массы $\mathrm{CaO}$ и $\mathrm{CO}_{2}$, кг/моль; $\mathrm{m}_{\mathrm{t}}$ и $\mathrm{m}_{0}$ - текущая и исходная массы образца доломита, кг; с - константа, характеризующая массовую долю реагирующих компонентов в исходном отожженном доломите, значение которого установлено на предыдущих стадиях исследования $(\mathrm{c}=0.57)$.

Константа скорости брутто-реакции записывается в форме аррениусовского уравнения для конкретного материала. На предыдущих этапах работы было показано, что для рассматриваемого доломита она может рассчитываться как:

$$
k_{p}=6 \cdot 10^{-3} \cdot \exp (-29600 / R \cdot T),
$$

где $\mathrm{R}$ - универсальная газовая постоянная, кг $\mathrm{c}^{-2} \cdot \mathrm{K}^{-1} \cdot$ моль $^{-1}$.

Таким образом, в рамках предложенной модели закон сохранения массы (3), может быть заменен рекуррентным балансовым матричным соотношением вида (8), в котором і-й элемент матрицы $\mathbf{M}$ рассчитывается с учетом убыли массы газовой фазы. Принимая во внимание, что $d m=d x \cdot\left(\mu_{\mathrm{CO}_{2}} \cdot c \cdot m_{0} / \mu_{\mathrm{CaO}}\right)$, можно получить

$$
M_{i}^{k}=\left(\frac{\eta_{0} \cdot k_{p, i}^{k} \cdot \mu_{\mathrm{CO}_{2}} \cdot c \cdot m_{0}}{\mu_{\mathrm{CaO}}}\right) \cdot\left(1-\frac{x_{i}^{k}}{x_{u}}\right),
$$

где $\eta_{0}-$ массовая доля $\mathrm{CO}_{2}$ вначале эксперимента; $k_{p, i}^{k}-$ константа скорости процесса для $i$-й ячейки на $k-$ м рекуррентном шаге, $\mathrm{c}^{-1} ; i-$ й ячейки на $k-\mathrm{M}$ рекуррентном шаге.

Необходимо подчеркнуть то обстоятельство, что модель (4)-(12) позволяет рассмотреть фильтр очистки как объект с распределенными и нестационарными характеристиками. Нестационарный характер процессов в первую очередь связан с прогревом слоя. Для моделирования процессов переноса теплоты используется та же вычислительная схема, что была использована нами ранее для описания нестационарного тепломассопереноса в цилиндрическом пиролизном реакторе.

Отметим также, что границами области моделирования являются наружные поверхности цилиндров с радиусами $\mathrm{R}_{1}$ и $\mathrm{R}_{2}$ (рис. 4). Через наружную поверхность подается газ со скоростью $\mathrm{U}_{0}$, а поверхность внутреннего цилиндра рассматривается как поглощающая поверхность (граничное условие первого рода).

\section{АНАЛИЗ РЕЗУЛЬТАТОВ}

На рисунке 5 представлены результаты расчетно-экспериментального исследования поглощения $\mathrm{CO}_{2}$ доломитовым сорбентом. Данные натурного эксперимента были получены следующим образом: через слой доломита пропускали непрерывный поток пиролизного газа с постоянным значением содержания $\mathrm{CO}_{2}$. Через определенное время после начала процесса подачу газа приостанавливали и по приращению массы навески определяли количество $\mathrm{CO}_{2}$, которое было связано сорбентом за это время. Для каждого опыта отбиралась новая навеска материала.

Для каждого времени процесса (60, $120, \ldots, 420$ c) опыт проводили трижды (результаты усредняли). Таким образом были получены данные по динамике поглощения $\mathrm{CO}_{2}$ дисперсным доломитом. В таблице 1 представлены результаты натурного эксперимента.

Изменение объемной доли $\mathrm{CO}_{2}$ в пиролизном газе при его прохождении через слой обожженного доломита ${ }^{6}$

\begin{tabular}{|c|c|c|c|c|c|c|c|c|}
\hline $\begin{array}{c}\text { Время процесса, } \\
\text { сек }\end{array}$ & 0 & 60 & 120 & 180 & 240 & 300 & 360 & 420 \\
\hline $\begin{array}{c}\text { массовая доля } \\
\mathrm{CO}_{2} \eta\end{array}$ & 0.15 & 0.145 & 0.134 & 0.06 & 0.012 & 0.009 & 0.002 & 0 \\
\hline
\end{tabular}


Результаты предложенного эксперимента в принципе должны быть эквивалентны многократному пропусканию очищаемого газа через засыпку. Поэтому балансовое соотношение (8) было модифицировано таким образом, чтобы масса газа, удаляемого из последней (внутренней) ячейки за время $\Delta \mathrm{t}$, поступала в первую ячейку. Таким образом, для рассматриваемого случая

$$
\mathbf{G}_{1}^{k}=p_{n} \cdot \mathbf{S}_{n}^{k},
$$

где нижний индекс отвечает за пространственное положение ячейки, a верхний - за номер временного перехода.

На рисунке 5 представлено сравнение результатов экспериментального и расчетного исследования массового содержания $\mathrm{CO}_{2}$ в газовом потоке. Засыпка при моделировании была представлена состоящей из $\mathrm{n}=12$ цилиндрических слоев.

Таким образом, для проведения численных экспериментов были приняты $\Delta \mathrm{t}=0.1$ с и $\Delta \mathrm{r}=0.01 \mathrm{м}$.

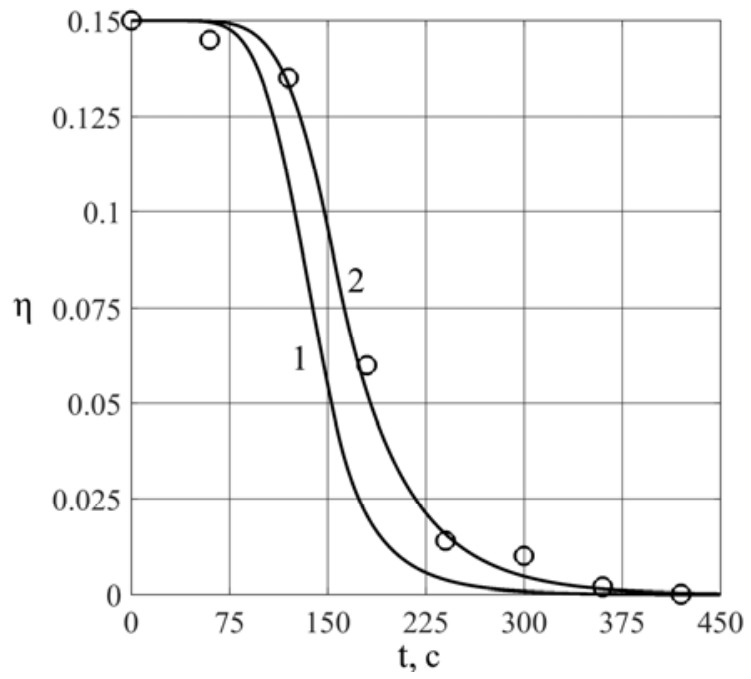

маркеры - экспериментальные значения, линии - расчетные значения.

1 - для внутренней ячейки фильтра (i=n); 2 - для внешней ячейки фильтра (i=1)

Рис. 5. Изменение во времени массовой доли $\mathrm{CO}_{2}$ в газовом агенте. ${ }^{7}$
На рисунке 6 представлены расчетные распределения температуры в отдельные моменты времени прогрева слоя.

Перед началом эксперимента навеска доломита выдерживалась в сухожаровом шкафу, поэтому начальная температура материала была порядка $100{ }^{\circ} \mathrm{C}$.

Показаны (рис. 6) распределения температур для двух близких моментов
Как видно из результатов расчета (рис. 5) изменение массового содержания $\mathrm{CO}_{2}$ в различных радиальных позициях внутри слоя происходит по-разному. Во внутренней ячейке слоя процесс ожидаемо завершается быстрее (линия 1), а во внутренней - с некоторым опозданием (линия 2), которое строго меньше времени однократно прохождения всего газа через засыпку.

Поскольку количество поглощенного газа определялась косвенным образом, и для его расчета проводилось определение изменения массы всей навески, то следует констатировать, что данные натурного эксперимента в целом хорошо согласуются с данными расчета, практически везде оставаясь между линиями 1 и 2. При этом для проверки адекватности модели в дальнейшем следует проводить измерения локальных параметров. времени на стадии прогрева, когда целевой процесс практически не идет.

Видно, что ячейки снаружи слоя, которые ближе к источнику горячего воздуха, прогреваются быстрее, хотя разница между температурами

крайних ячеек не велика (несколько градусов), 
и сохраняется более-менее постоянной. На рисунке 7 показано изменение средней температуры слоя со временем. Как видно, температура меняется почти линейно в процессе прогрева слоя, что, вероятно, объясняется высокой теплопроводностью слоя и интенсивным теплообменом газ- твердое. Однако, несмотря на отсутствие больших температурных градиентов в слое, имеет место заметное расхождение в степени завершенности реакции в наружных и внутренних ячейках слоя, что необходимо иметь в виду при расчете систем очистки.

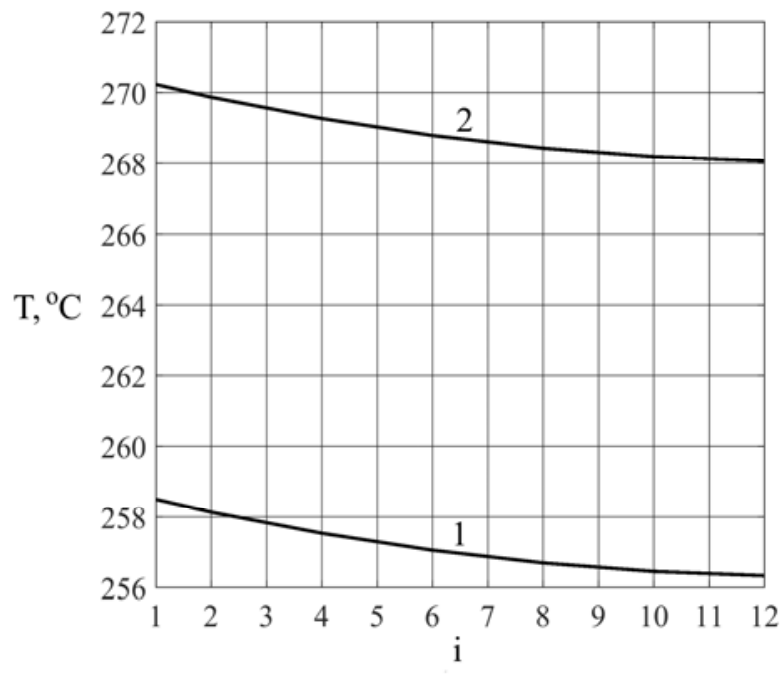

1 - для момента времени $t=45$ мин; 2 - для момента времени $t=50$ мин

Рис. 6. Распределение температуры сыпучего материала по ячейкам слоя. ${ }^{8}$

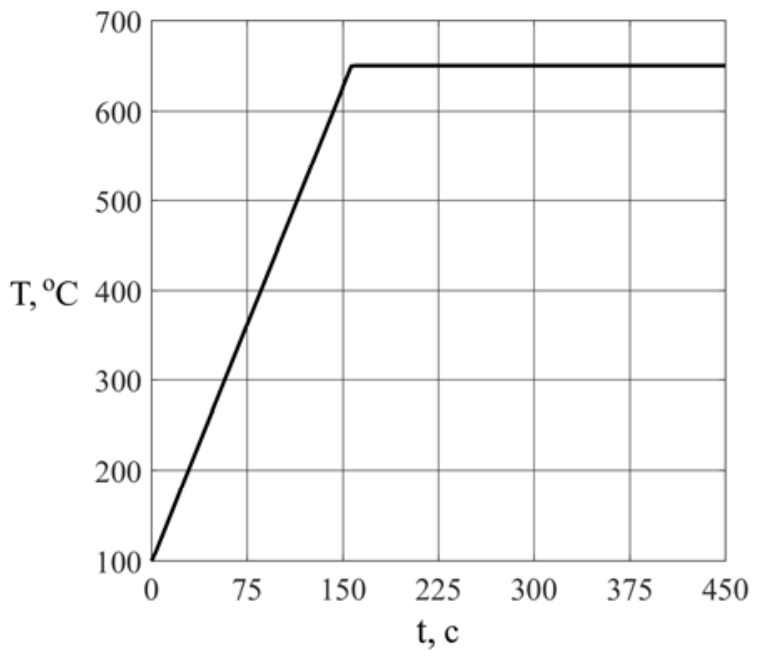

Рис. 7. Зависимость средней температуры твердой фазы от времени (температура подаваемого газового агента $\left.650{ }^{\circ} \mathrm{C}\right) .{ }^{9}$

\section{ВЫВОДЫ}

1. В работе выполнено построение и проведена верификация математической модели функционирования слоевого сорбционного фильтра с радиальноаксиальной схемой движения генераторного газа через засыпку дисперсного доломита. Для построения одномерной математической модели процесса с дискретным пространством и временем использован математический аппарат теории счетных цепей Маркова. При этом значения элементов переходной матрицы, а также векторов источников и стоков поставлены в соответствие физико-химическим параметрам процесса. Тот факт, что основное балансовое уравнение модели формируется на каждом расчетном шаге с учетом текущих параметров процесса, делает предложенную модель нелинейной.

2. Одним из наиболее значимых результатов исследования является то, что 
выполнена параметрическая идентификация предложенной модели, которая наделяет ее прогностическими свойствами. Выполнена проверка прогностической эффективности и адекватности модели через сопоставление получаемых прогнозов с результатами натурного принципиально независимого от схемы моделирования эксперимента.

3. Наиболее общий вывод, определяемый результатами исследования, состоит в том, что в ходе работы предложен научно обоснованный подход к решению проблемы повышения достоверности описания и надежности прогнозирования технологических процессов в слоевом фильтре. Указанный подход, основанный на построении математических моделей технологических процессов в слое, в которых фильтр рассматривается как система с распределенными характеристиками, а расчет ведется по локальным потенциалам обмена между частицами и газом.

\section{ACKNOWLEDGEMENTS}

Исследование выполнено при финансовой поддержке Российского фонда фундаментальных исследований и Ивановской области в рамках научного проекта № 20-48-370001

\section{APPENDIX 1 (ПРИЛОЖЕНИЕ 1)}

${ }^{1}$ Fig. 1. Schematic diagram of a pyrolysis reactor and connection of bed filters for cleaning pyrolysis gases (a), photos of a general view of an experimental sample of a bed cleaning filter with radial-axial movement of gases (b) and a perforated cassette (c).

${ }^{2}$ Fig. 2. The general scheme of the laboratory setup for the study of the hydraulic characteristics of the dolomite bed in the bed filter.

${ }^{3}$ Fig. 3. To the description of the experiment on measuring statistical pressure in the bed: a diagram of a radial-axial reactor in the section with static pressure measurement points ((a) - top view; (b) - side view), (c) - measurement results.

${ }^{4}$ Fig. 4. Calculation scheme of the mathematical cell model.

${ }^{5,6}$ Table 1. Change in the volume fraction of $\mathrm{CO}_{2}$ in pyrolysis gas during its passage through a bed of burnt dolomite.

${ }^{7}$ Fig. 5. Time change in the mass fraction of $\mathrm{CO}_{2}$ in the gas agent.

${ }^{8}$ Fig. 6. Temperature distribution of the bulk material across the bed cells.

${ }^{9}$ Fig. 7. Dependence of the average temperature of the solid phase on time (temperature of the supplied gas agent $650{ }^{\circ} \mathrm{C}$ ).

\section{Литература (References)}

[1] Fortov V.E., Popel' O.S. The Current Status of the Development of Renewable Energy Sources Worldwide and in Russia. Thermal Engineering, 2014, vol. 61, no.6, pp. 389-398.

[2] De S., Agarwal A.K., Moholkar V.S., Bhaskar T. Coal and Biomass Gasification. Recent Advances and Future Challenges. Springer Nature, Singapore Pte Ltd., 2018, 521 p.

[3] Afanas'eva O.V., Mingaleeva G.R. Energy Efficiency of Small Coal-Fired Power Plants as a Criterion of Their Wide Applicability. Solid Fuel Chemistry, 2009, vol. 43, no. 1, pp. 55-59.

[4] Hu G., Xu S., Li S., Xiao C., Liu S. Steam Gasification of Apricot Stones with Olivine and Dolomite as Downstream Catalysts. Fuel Processing Technology, 2006, Vol. 87, No. 5, p. 375-382.

[5] Olszak-Humienik M., Jablonski M. Thermal Behavior of Natural Dolomite. Journal of Thermal Analysis and Calorimetry, 2015, vol. 119, pp. 2239-2248.

[6] Dobrego K.V. Makrokineticheskie Modeli Termicheskogo Razlozheniya Dolomita dlya Rascheta Sorbtsionnykh Sistem Gazogeneratorov [Dolomite Thermal-Decomposition Macrokinetic Models for Evaluation of the Gasgenerators Sorbent Systems]. Energetika. Proceedings of CIS Higher Education Institutions and Power Engineering Associations, 2015, no. 5, pp. 51-59. (In Russian).

[7] Rat'ko A.I., Ivanets A.I., Kulak A.I., Morozov E.A., Sakhar I.O. Thermal Decomposition of Natural Dolomite. Inorganic Materials, 2011, vol. 47, no.12, pp. 1372-1377.

[8] Maitra S., Chowdhury A., Das H., Pramanik Ms. Effect of Compaction on the Kinetics of Thermal Decomposition of Dolomite under NonIsothermal Condition. Journal of Materials Science, 2005, vol. 40, pp. 4749-4751.

[9] Gunasekaran S., Anbalagan G. Thermal Decomposition of Natural Dolomite. Bulletin of Materials Science, 2007, vol. 30, pp. 339-344.

[10] Lee D., An Apparent Kinetic Model for the Carbonation of Calcium Oxide by Carbon Dioxide. Chemical Engineering Journal, 2004, vol. 100, pp. 71-77.

[11] Salaudeen S.A., Acharya B., Dutta A., CaOBased CO2 Sorbents: a Review on Screening, Enhancement, Cyclic Stability, Regeneration and Kinetics Modeling. Journal of $\mathrm{CO}_{2}$ Utilization, 2018, vol. 23, pp. 179-199.

[12] Fedunik-Hofman L., Bayon Sandoval A., Donne S. Kinetics of Solid-Gas Reactions and Their Application to Carbonate Looping Systems. Energies. 2019, vol. 12. , pp. 1-35.

[13] Bhagiyalakshmi, M.; Hemalatha, P.; Ganesh, M.; Mei, P.M.; Jang, H.T. A Direct Synthesis of Mesoporous Carbon Supported MgO Sorbent for 
$\mathrm{CO}_{2}$ Capture. Fuel, 2011, vol. 90, pp.1662-1667

[14] Li Z., Fang F., Tang X., Cai N., Effect of Temperature on the Carbonation Reaction of $\mathrm{CaO}$ with $\mathrm{CO}_{2}$. Energy\&Fuels, 2012, vol. 26, pp. 2473-2482

[15] van der Hoef M.A., Ye M., van Sint Annaland M., Andrews IV A.T., Sundaresan S., Kuipers J.A.M. Multiscale Modeling of Gas-Fluidized Beds. Advances in Chemical Engineering, 2006, vol. 31, pp. 65-149.

[16] Deen N.G., van. Sint Annaland M., van der Hoef M.A., Kuipers J.A.M. Review of Discrete Particle Modeling of Fluidized Beds. Chem. Eng. Science, 2007, vol. 62, pp. 28-44.

[17] Dai Q., Chen C., Qi H. Influence of Meso-Scale Structures on Drag in Gas-Solid Fluidized Beds. Powder Technology. 2016, vol. 288, pp. 87-95.

[18] Dehling H.G., Hoffmann A.C., Stuut H.W. Stochastic Models for Transport in a Fluidized Bed. SIAM J. Appl. Math, 1999, Vol.60, pp. 337-358.

[19]Zhukov V.P., Belyakov A.N. Simulation of Combined Heterogeneous Processes Based on

\section{Сведения об авторах.}
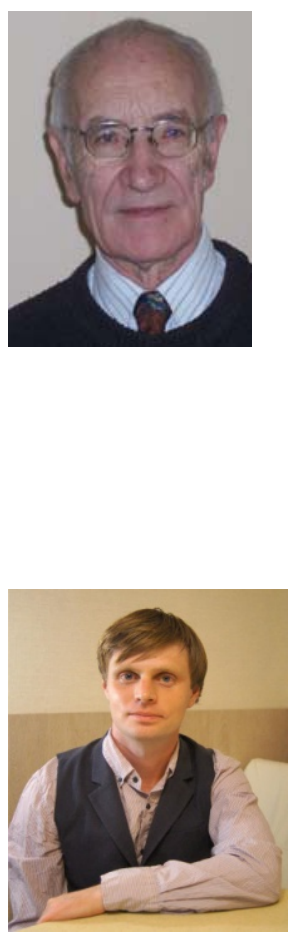

\section{Малько Михаил Вла-}

димирович, кандидат физико-математических наук, ведущий научный сотрудник Института энергетики НАН Беларуси, Республика Беларусь. Область научных интересов:

возобновляемая энергетика, получение биотоплив.

E-mail:

mikhailvm@bas-net.by

Митрофанов Андрей

Васильевич, доктор технических наук, Ивановский государственный энергетический университет, Российская Федерация. Область научных интересов: процессы в кипящем слое. E-mail: and2mit@mail.ru
Discrete Models of the Boltzmann Equation. Theor. Found. Chem. Eng., 2017, vol. 51, pp. 8893.

[20] Bobkov S.P. Simulation of Basic Transfer Processes Using Cellular Automata. Russian Journal of Chemistry and Chemical Technology. 2009, vol. 3, no.52, pp.109-114.

[21] Loitsyaskiy L.G. Mechanics of Liquids and Gases, Begell House, New York and Wallingford, 6th ed, 1995, $961 \mathrm{p}$.

[22] Tamir A. Applications of Markov Chains in Chemical Engineering, Elsevier, Amsterdam, 1998, $604 \mathrm{p}$.

[23] Hartman M., Trnka O., Vesely V., Svoboda K. Predicting the Rate of Thermal Decomposition of Dolomite. Chemical Engineering Science, 1996, vol. 51, no. 23, pp. 5229-5232.

[24] Lee D., An Apparent Kinetic Model for the Carbonation of Calcium Oxide by Carbon Dioxide, Chemical Engineering Journal, 2004, vol 100, pp. 71-77.

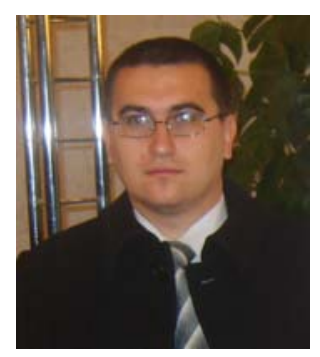

Василевич Сергей Владимирович, кандидат технических наук, доцент Белорусской государственной академии авиации, Республика Беларусь. Область научных интересов: возобновляемая энергетика, получение биотоплив. E-mail: svasilevich@yandex.ru

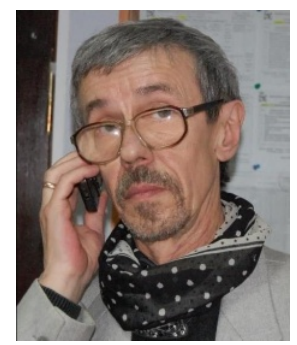

Мизонов Вадим Евгеньевич, доктор технических наук, Ивановский государственный энергетический университет, Российская Федерация. Область научных интересов: применение теории цепей Маркова в химической инженерии. E-mail: mizonov46@mail.ru 